\title{
VALORES HEMATOLÓGICOS, BILIRRUBINEMIA Y ACTIVIDAD ENZIMÁTICA SÉRICA EN CABALLOS PERUANOS DE PASO DEL VALLE DE LURÍN, LIMA
}

\author{
Hematologic Values, Bilirubin and EnzymeActivities in Serum of Peruvian \\ Paso Horses from Lurin Valley, Lima
}

Henry Díaz G. ${ }^{1,2}$, César Gavidia Ch. ${ }^{1,3}$, Olga Li E. ${ }^{4}$, Alfredo Tió G. ${ }^{5}$

\section{ResUMen}

\begin{abstract}
Los parámetros hematológicos y bioquímicos sanguíneos son herramientas útiles en la clínica médica equina, pero su utilidad al servicio del Caballo Peruano de Paso es limitado ante la ausencia de valores de referencia. El estudio se realizó para determinar los valores hemato-bioquímicos en ejemplares aparentemente sanos. Se estudiaron 49 animales clasificados según sexo [machos $(n=17)$ y hembras $(n=30)$ ] y edad [jóvenes $(n=23)$ $\mathrm{y}$ adultos $(\mathrm{n}=26)$ ]. El perfil hematológico (recuento total de eritrocitos, hematocrito, concentración de hemoglobina, volumen corpuscular medio, concentración de hemoglobina corpuscular media, recuento total de leucocitos, neutrófilos abastonados, neutrófilos segmentados, eosinófilos, monocitos, linfocitos, relación neutrófilo/linfocito y el recuento de plaquetas) y bioquímico (bilirrubina total, bilirrubina directa, bilirrubina indirecta, ALT, AST, ALP, GGT y CK) se estimaron mediante analizadores automáticos. Se determinó el efecto del sexo y la edad sobre los valores obtenidos. El sexo influyó significativamente el recuento de monocitos y la actividad de la enzima CK, y la edad afectó la concentración de hemoglobina, volumen corpuscular medio, concentración de hemoglobina corpuscular media, linfocitos, relación neutrófilo/linfocito, ALT, AST, bilirrubina total y bilirrubina indirecta $(\mathrm{p}<0.05)$.
\end{abstract}

Palabras clave: Caballo Peruano de Paso, parámetros hemato-bioquímicos, perfil fisiológico

\section{ABSTRACT}

The hematological and blood biochemical parameters are useful tools for equine medical practice but their usefulness for the Peruvian Paso Horse is limited by the absence of reference values. The present study was conducted to determine hemato-biochemical values in apparently healthy horses. Forty nine animals were evaluated and they were

\footnotetext{
${ }^{1}$ Laboratorio de Medicina Veterinaria Preventiva, ${ }^{4}$ Laboratorio de Patología Clínica y Biología Molecular, Facultad de Medicina Veterinaria, Universidad Nacional Mayor de San Marcos, Lima

${ }^{2}$ E-mail: gen_etico@yahoo.es

${ }^{3}$ E-mail: cmgavidia@yahoo.com

${ }^{5}$ Práctica privada
} 
classified according to sex [males $(n=17)$, females $(n=30)$ ] and age [young $(n=23)$, adults $(n=26)$ ]. The hematological (red blood cell count, hematocrit, hemoglobin concentration, mean corpuscular volume, mean corpuscular hemoglobin concentration, white blood cell count, band neutrophils, segmented neutrophils, eosinophils, monocytes, lymphocytes, neutrophil/lymphocyte ratio and platelet count) and the biochemical (total bilirubin, direct bilirubin, indirect bilirubin, ALT, AST, ALP, GGT and CK) profiles were estimated by means of automated analyzers. The effect of sex and age was evaluated. Sex influenced the number of monocytes and CK activity, and age influenced hemoglobin concentration, mean corpuscular volume, mean corpuscular hemoglobin concentration, lymphocytes, neutrophil/lymphocyte ratio, ALT, AST, total bilirubin and indirect bilirubin $(\mathrm{p}<0.05)$.

Key words: Peruvian Paso Horse, hemato-biochemical parameters, physiological profile

\section{INTRODUCCIÓN}

El Caballo Peruano de Paso ha permanecido genéticamente aislado por varios siglos (Rodríguez-Gallardo et al., 1992), tiempo en el cual la idiosincrasia de sus criadores, la geografía de la región y su utilidad como animal viajero, medio de transporte y herramienta de trabajo en la agricultura definirían sus actuales características (Risso, 1994).

La utilidad de la patología clínica en medicina equina se extiende a la formulación del pronóstico, monitoreo de tratamientos, implementación de medidas preventivas, cumplimiento de regulaciones gubernamentales para el tránsito de animales y particularmente a la evaluación del desempeño físico (Rossdale and Ricketts, 1980). La correcta interpretación de los resultados del análisis clínico requiere de valores de referencia que procedan de la población de origen debido a la influencia de importantes variables relacionadas al manejo, ambientales o genéticas (Lording, 2008). Así, cada raza caballar expresará características fenotípicas y metabólicas específicas relacionadas con el tipo de actividad que desarrolla (Lacerda $e t$ al., 2006).

En las últimas décadas, el manejo clínico y nutricional diferenciado ha propuesto como marcadores objetivos de la fisiología y del nivel de entrenamiento, los cambios fi- siológicos, hematológicos y bioquímicos observados en razas equinas tras competencias de velocidad (Kedzierski y Bergero, 2006), cabalgatas de resistencia (Andrews et al., 1995), competencias de polo (Craig et al., 1985), de rodeo (Pérez et al., 1997) y salto (Gómez et al., 2004).

El presente estudio tuvo por objetivo caracterizar parte del perfil fisiológico en un grupo definido de Caballos Peruanos de Paso ante la carencia de valores referenciales comparables de parámetros involucrados en el diagnóstico de patologías frecuentemente detectadas en la especie equina como trastornos musculares, hepáticos, anemia y policitemia (Coppo y Mussart, 2000).

\section{Materiales y Métodos}

Se trabajó con 49 equinos de la raza Caballo Peruano de Paso procedentes de cuatro criaderos ubicados en el departamento de Lima. La edad de los animales fue de $4.6 \pm 3.7$ años (rango de 4 meses a 21 años). La muestra de animales comprendió 17 machos enteros, 30 hembras y 2 capones. La distribución de estos animales por edad correspondió a 23 jóvenes (menor o igual a 3 años) y 26 adultos (mayor de 3 años).

Se hizo una evaluación clínica inicial para constatar su aparente buen estado de 
Cuadro 1. Valores hematológicos medios, máximos, mínimos y desvío estándar de la serie roja (glóbulos rojos [GR], hemoglobina $[\mathrm{Hb}]$, hematocrito [Ht], volumen corpuscular medio [VCM] y concentración de hemoglobina corpuscular media $[\mathrm{CHCM}])$ en equinos de la raza Caballo Peruano de Paso criados en el valle de Lurín (Lima)

\begin{tabular}{clccccc}
\hline \multicolumn{2}{c}{ Grupo } & $\begin{array}{c}\text { GR } \\
\left(\mathrm{x} 10^{6} / \mu \mathrm{L}\right)\end{array}$ & $\begin{array}{c}\mathrm{Hb} \\
(\mathrm{g} / \mathrm{dL})\end{array}$ & $\begin{array}{c}\mathrm{Ht} \\
(\%)\end{array}$ & $\begin{array}{c}\mathrm{VCM} \\
(\mathrm{fL})\end{array}$ & $\begin{array}{c}\text { CHCM } \\
(\mathrm{g} / \mathrm{dL})\end{array}$ \\
\hline \multirow{2}{*}{ Sexo } & Macho & $8.7 \pm 0.9$ & $14.2 \pm 1.6$ & $41.9 \pm 4.8$ & $48.4 \pm 4.1$ & $33.9 \pm 0.7$ \\
& Hembra & $8.2 \pm 0.9$ & $13.7 \pm 1.4$ & $40.0 \pm 4.3$ & $48.1 \pm 6.3$ & $34.2 \pm 0.7$ \\
\multirow{2}{*}{ Edad } & Joven & $8.5 \pm 0.9$ & $13.4 \pm 1.5^{\mathrm{a}}$ & $39.7 \pm 4.9$ & $46.5 \pm 2.7^{\mathrm{a}}$ & $33.9 \pm 0.7^{\mathrm{a}}$ \\
& Adulto & $8.1 \pm 0.8$ & $14.3 \pm 1.4^{\mathrm{b}}$ & $41.7 \pm 4.1$ & $50.1 \pm 6.7^{\mathrm{b}}$ & $34.4 \pm 0.7^{\mathrm{b}}$ \\
\hline \multirow{2}{*}{ Total } & & $8.3 \pm 0.9$ & $13.9 \pm 1.5$ & $40.7 \pm 4.6$ & $48.4 \pm 5.5$ & $34.1 \pm 0.7$ \\
& & $(6.5-10.4)$ & $(11.4-17.1)$ & $(33.3-52.0)$ & $(20.7-57.8)$ & $(32.8-35.6)$ \\
\hline
\end{tabular}

${ }^{a, b}$ Superíndices diferentes dentro de columnas son estadísticamente diferentes $(p<0.05)$

salud. Las muestras se colectaron por las mañanas después de 20 minutos de reposo posterior a su sujeción. La sangre se obtuvo por punción de la vena yugular en tubos de vidrio con vacío (Vacutainer®) conteniendo EDTA para el análisis hematológico y sin anticoagulante para el análisis bioquímico. $\mathrm{El}$ suero se obtuvo por centrifugación ( $2500 \mathrm{rpm}$, 15 minutos), manteniéndose en refrigeración hasta la realización del análisis.

El perfil hematológico (recuento total de eritrocitos, hematocrito, concentración de hemoglobina, volumen corpuscular medio, concentración de hemoglobina corpuscular media, recuento total de leucocitos, fórmula leucocitaria, relación neutrófilo/linfocito y recuento de plaquetas) se determinó mediante el analizador hematológico automático Sysmex SF-3000 (Sysmex Corporation, Japón). El perfil bioquímico (bilirrubina total, directa e indirecta, ALT, AST, ALP, GGT y CK) se determinó con métodos espectrofotométricos utilizando kits diagnósticos comerciales y el analizador automático Hitachi 911 (Roche Diagnostics Corporation, USA).
Las variables se sometieron a un test de normalidad (Shapiro Wilk) antes del análisis estadístico descriptivo Los resultados se expresaron en tablas de frecuencia estratificadas para comparar el efecto del sexo y la edad, bajo un nivel de significancia de 0.05 mediante las pruebas " $t$ " de Student y U de Mann Whitney según la naturaleza de cada variable, empleando el paquete estadístico STATA 10.0. Los valores de los capones no se incluyeron en los análisis por efecto del sexo.

\section{Resultados}

El test de normalidad reveló que los parámetros hematológicos analizados presentaron una distribución normal a excepción de los neutrófilos abastonados, monocitos y eosinófilos. En cambio, los parámetros bioquímicos no presentaron una distribución normal a excepción de la bilirrubina directa y la AST.

En el Cuadro 1 se muestran los valores hematológicos de la serie roja. No se observó diferencias estadísticas en relación al sexo; 
Cuadro 2. Valores leucocitarios medios y desvío estándar (Glóbulos blancos [GB], abastanados [Ab], segmentados [Seg], eosinófilos [Eos], monocitos [Mon], linfocitos [Lin]) en equinos de la raza Caballo Peruano de Paso criados en el valle de Lurín (Lima)

\begin{tabular}{|c|c|c|c|c|c|c|}
\hline Grupo & $\begin{array}{c}\text { GB } \\
(/ \mu \mathrm{L})\end{array}$ & $\begin{array}{c}\mathrm{Ab} \\
(/ \mu \mathrm{L})\end{array}$ & $\begin{array}{l}\text { Seg } \\
(/ \mu \mathrm{L})\end{array}$ & $\begin{array}{c}\text { Eos } \\
(/ \mu \mathrm{L})\end{array}$ & $\begin{array}{l}\text { Mon } \\
(/ \mu \mathrm{L})\end{array}$ & $\begin{array}{l}\text { Lin } \\
(/ \mu \mathrm{L})\end{array}$ \\
\hline \multicolumn{7}{|l|}{ Sexo } \\
\hline Macho & $8635 \pm 2310$ & $0 \pm 0$ & $4233 \pm 1232$ & $163 \pm 203$ & $249 \pm 147^{?}$ & $3985 \pm 1575$ \\
\hline Hembra & $9233 \pm 1365$ & $2 \pm 13$ & $4429 \pm 950$ & $197 \pm 174$ & $173 \pm 141^{?}$ & $4430 \pm 1261$ \\
\hline \multicolumn{7}{|l|}{ Edad } \\
\hline Joven & $9443 \pm 1992$ & $0 \pm 0$ & $4179 \pm 1116$ & $114 \pm 107 *$ & $195 \pm 143$ & $4953 \pm 1315^{*}$ \\
\hline Adulto & $8503 \pm 1440$ & $5 \pm 20$ & $4523 \pm 952$ & $240 \pm 211 *$ & $207 \pm 148$ & $3523 \pm 1119^{*}$ \\
\hline Total & $8944 \pm 1767$ & $3 \pm 15$ & $4361 \pm 1036$ & $181 \pm 181$ & $202 \pm 44$ & $4195 \pm 1402$ \\
\hline
\end{tabular}

Cuadro 3. Valores de la relación segmentados/linfocitos y recuento plaquetario en equinos de la raza Caballo Peruano de Paso criados en el valle de Lurín (Lima)

\begin{tabular}{llcc}
\hline & & $\begin{array}{c}\text { Relación } \\
\text { segmentados/linfocitos }\end{array}$ & $\begin{array}{c}\text { Plaquetas } \\
(/ \mu \mathrm{L})\end{array}$ \\
\hline \multirow{2}{*}{ Sexo } & Macho & $1.1 \pm 0.4$ & $175235 \pm 38507$ \\
& Hembra & $1.1 \pm 0.5$ & $184933 \pm 46668$ \\
\multirow{2}{*}{ Edad } & Joven & $0.9 \pm 0.3^{\mathrm{a}}$ & $186652 \pm 34762$ \\
& Adulto & $1.4 \pm 0.4^{\mathrm{b}}$ & $179461 \pm 51215$ \\
\hline \multirow{2}{*}{ Total } & Media & $1.2 \pm 0.5$ & $182836 \pm 43967$ \\
& Rango & $(0.6-2.2)$ & $(85000-261000)$ \\
\hline
\end{tabular}

${ }^{a, b}$ Superíndices diferentes dentro de columnas son estadísticamente diferentes $(p<0.05)$

sin embargo, las concentraciones de hemoglobina, el VCM y el CHCM fueron significativamente superiores $(\mathrm{p}<0.05)$ en equinos adultos en comparación con jóvenes.

Los valores de la serie blanca se muestran en los cuadros 2 y 3. Se observó diferencia estadística entre sexos en el recuento de monocitos a favor de los machos ( $\mathrm{p}<0.05$ ), mientras que se encontró mayor cantidad de eosinófilos en animales adultos y de linfocitos en animales jóvenes ( $p<0.05$, Cuadro 2). Asimismo, la relación segmentados/linfocitos fue estadísticamente favorable para animales adultos ( $\mathrm{p}<0.05$, Cuadro 4$)$.

En los cuadros 4 y 5 muestran los valores de bioquímica sanguínea. Se observó diferencia por efecto del sexo en la actividad de la CK ( $\mathrm{p}<0.05)$. Asimismo, la actividad de la ALT y AST fue superior en animales jóvenes, y de bilirrubina total e indirecta en animales adultos $(\mathrm{p}<0.05)$. 
Cuadro 4. Valores medios, máximos, mínimos y desvío estándar de la actividad enzimática sérica en equinos de la raza Caballo Peruano de Paso criados en el valle de Lurín (Lima)

\begin{tabular}{lcccccc}
\hline & $\begin{array}{c}\text { ALT } \\
(\mathrm{U} / \mathrm{L})\end{array}$ & $\begin{array}{c}\text { AST } \\
(\mathrm{U} / \mathrm{L})\end{array}$ & $\begin{array}{c}\text { ALP } \\
(\mathrm{U} / \mathrm{L})\end{array}$ & $\begin{array}{c}\text { GGT } \\
(\mathrm{U} / \mathrm{L})\end{array}$ & $\begin{array}{c}\text { CK } \\
(\mathrm{U} / \mathrm{L})\end{array}$ \\
\hline Sexo & & & & & & \\
& Macho & $13.6 \pm 3.3$ & $327 \pm 85$ & $60 \pm 21$ & $8.0 \pm 1.5$ & $406 \pm 175^{?}$ \\
& Hembra & $11.8 \pm 3.0$ & $323 \pm 63$ & $62 \pm 21$ & $8.0 \pm 4.0$ & $466 \pm 85^{?}$ \\
\multirow{2}{*}{ Edad } & & & & & \\
& & & & & & \\
& Joven & $14.0 \pm 3.4^{*}$ & $356 \pm 68^{*}$ & $62 \pm 20$ & $8.0 \pm 1.7$ & $433 \pm 113$ \\
& Adulto & $10.8 \pm 2.3^{*}$ & $290 \pm 61^{*}$ & $58 \pm 22$ & $8.1 \pm 4.2$ & $442 \pm 145$ \\
\hline \multirow{2}{*}{ Total } & & $12.3 \pm 3.3$ & $321 \pm 72$ & $60 \pm 21$ & $8.0 \pm 3.2$ & $438 \pm 129$ \\
& & $(6-23)$ & $(212-519)$ & $(33-122)$ & $(4-27)$ & $(219-955)$ \\
\hline
\end{tabular}

ALT, Alanina aminotransferasa; AST, Aspartato aminotransferasa; ALP, Fosfatasa alcalina; GGT, Gammaglutamiltransferasa; CK, Creatinkinasa

?, $\mathrm{p}<0.05$ en relación al sexo

*, p $<0.05$ en relación a la edad

Cuadro 5. Valores medios, máximos, mínimos y desvío estándar de la bilirrubinemia en equinos de la raza Caballo Peruano de Paso criados en el valle de Lurín (Lima)

\begin{tabular}{llccc}
\hline & & $\mathrm{BT}$ & $\mathrm{BD}$ & $\mathrm{BI}$ \\
$(\mathrm{mg} / \mathrm{dL})$ & $(\mathrm{mg} / \mathrm{dL})$ & $(\mathrm{mg} / \mathrm{dL})$ \\
\hline Sexo & & & \\
& Macho & $1.9 \pm 1.5$ & $0.4 \pm 0.1$ & $1.5 \pm 1.4$ \\
& Hembra & $1.9 \pm 1.0$ & $0.4 \pm 0.1$ & $1.5 \pm 1.0$ \\
\multirow{2}{*}{ Edad } & & & \\
& Joven & $1.4 \pm 0.7^{*}$ & $0.4 \pm 0.1$ & $1.0 \pm 0.6^{*}$ \\
& Adulto & $2.3 \pm 1.4^{*}$ & $0.4 \pm 0.1$ & $1.9 \pm 1.3^{*}$ \\
\hline \multirow{2}{*}{ Total } & & $1.9 \pm 1.2$ & $0.4 \pm 0.1$ & $1.5 \pm 1.1$ \\
& & $(0.6-7.0)$ & $(0.1-0.7)$ & $(0.4-6.3)$ \\
\hline
\end{tabular}

BT, Bilirrubina Total; BD, Bilirrubina Directa; BI, Bili rrubina Indirecta

*, $p<0.05$ en relación a la edad 


\section{Discusión}

La evaluación del tipo de distribución de las variables mediante el test de normalidad reveló semejanzas a la descrita para caballos de carrera (Blackmore y Brobst, 1981). Esto incluye considerar a los parámetros de la serie roja como variables de distribución normal y a la mayoría de los parámetros considerados en la estimación de la actividad sérica enzimática y bilirrubinemia como variables de distribución no normal (lognormal). La definición del tipo de distribución de una variable mejora la interpretación estadística, pero algunos autores obvian este paso asumiendo que todas sus variables siguen una distribución normal en base a sus propios antecedentes y a la influencia del tamaño de la muestra (Muñoz et al., 2001). Por lo tanto, la razón por la cual algunas variables podrían seguir una distribución diferente a las citadas es atribuible a la falta de antecedentes, al tamaño de la muestra y a la falta de aleatoriedad en la selección de los caballos.

Si se asume, según Lording (2008), que el rango de valores en el recuento de eritrocitos observado en razas ligeras o atléticas (sangre caliente) y pesadas o de tiro (sangre fría) es de 8.2 - 12.2 y $5.5-9.5$ (x $10^{6} /$ ? L), respectivamente, entonces el valor medio obtenido en este estudio $\left(8.3 \times 10^{6} \%\right.$ ?L) se encuentra en el extremo inferior del rango normal de valores definidos para caballos de sangre caliente y en el extremo superior del rango normal de valores definidos para caballos de sangre fría (Schalm et al., 1975). El mismo patrón fue observado para la concentración de hemoglobina y, en forma menos obvia, para el recuento total de leucocitos, excepto para el hematocrito y los índices eritrocíticos que de él dependen, probablemente debido a factores que determinan su inestabilidad como la excitación ante el manejo propio de algunos ejemplares.

El valor medio del recuento de eritrocitos es comparable con aquellos reportados de razas de sangre tibia o "warm blooded", como es el caso del Cuarto de Milla ( $8.26 \times 10^{6} /$ ? L), el Appaloosa ( $8.6 \times 10^{6} /$ ? ) y el Standardbred $\left(8.37 \times 10^{6} /\right.$ ? L) según Harvey y Hambright (1985), y el Criollo (8.59 x 106/?L) según Lacerda et al. (2006). Asimismo, en caballos Andaluz (actual Pura Sangre Español), un ancestro del Caballo Peruano de Paso, se reporta valores semejantes $(6.73 \mathrm{x}$ $10^{6} /$ ? L), aunque valores mayores en el recuento de leucocitos totales (Riber et al., 1995). Ambas condiciones también han sido descritas para el caballo Lipizzano en relación al ancestro común Andaluz (ÄebuljKadunc et al., 2002), dejando abierta la posibilidad de cambios fisiológicos generados por un proceso de adaptación.

A pesar de que el análisis estadístico no reflejó diferencias estadísticas por efecto del sexo en los parámetros hematológicos, se pudo corroborar la tendencia reportada en la literatura científica que los caballos machos intactos tienden a tener un recuento de glóbulos rojos, hematocrito y concentración de hemoglobina ligeramente mayor en comparación con las hembras (Deem, 2002). Por otro lado, un estudio desarrollado en el caballo Criollo sugiere que el valor medio del VCM de hembras es significativamente mayor al de machos como un efecto compensatorio a la menor cantidad de glóbulos rojos (Medeiros et al., 2006); sin embargo, esta tendencia no se observó en el presente estudio, posiblemente debido a que la edad de las hembras (4.6 años) era mayor que en los machos (3.2 años), y este factor afecta al VCM en forma directamente proporcional.

La edad afectó la concentración de hemoglobina $(13.4 \pm 1.5$ en jóvenes y $14.3 \pm$ 1.4 en adultos). Una explicación propuesta está referida al déficit de hierro que presentan los animales jóvenes en esta etapa de desarrollo (Bayly, 1987). El hematocrito no fue afectado por la edad, a diferencia de otros reportes en otras razas (Äebulj-Kadunc et al., 2002; Medeiros et al., 2006). Es posible que estas diferencias hubieran sido mayores de haberse trabajado con potrillos más jóvenes, toda vez que la hematopoyesis es menor en 
potrillos (Benjamín, 1991). En otro sentido, los índices eritrocíticos (VCM y CHCM) en animales adultos fueron significativamente mayores a los encontrados en los más jóvenes, similar a reportes en otras razas (Schalm et al., 1975).

Los adultos presentaron un significativo descenso en el recuento de linfocitos (4953/. L) en relación a los jóvenes (3523/ ?L), el cual es proporcional a la disminución en el número de leucocitos totales (9443 para jóvenes y 8503/? L para adultos), así como en la relación segmentados/linfocitos (0.9 para jóvenes y 1.4 para adultos). Estas mismas condiciones han sido descritas también en el caballo Lipizzano (Äebulj-Kadunc et al., 2002) y en otras razas caballares (Schalm et $a l ., 1975)$, de allí que algunos autores sugieren que esta dependencia con la edad en animales sanos puede contribuir a un descenso en su inmunocompetencia (McFarlane et al., 2001).

Las diferencias halladas en relación al recuento de eosinófilos entre jóvenes (1114/ ?L) y adultos (240/?L) se consideran, principalmente, como producto de un probable cambio en la exposición inmunológica en relación al parasitismo (Jain, 1993). Asimismo, la literatura científica señala que los neutrófilos en banda, basófilos y monocitos son células que en condiciones normales son muy poco frecuentes en sangre periférica, de modo que la variabilidad que se puede encontrar no se considera representativa (García, 2006). Por otro lado, el valor promedio del recuento plaquetario (182 836/? L) se encuentra dentro del rango normal de valores $(100000-400000 / ? 1)$, indistinto al sexo o edad de los animales (Schalm y Carlson, 1982).

El valor sérico de ALT $(12.27 \pm 3.25 \mathrm{U} / \mathrm{L})$ fue menor al $14 \pm 11 \mathrm{U} / \mathrm{L}$ definido por Kaneko et al. (1997), aunque los valores en ambos casos presentan una amplia variabilidad, de modo que estas diferencias no se consideran de importancia. Asimismo, tomando en cuenta que las enzimas sanguíneas de los potros son considerablemente mayores que en los adultos (Selvaraj et al., 2008), la diferencia hallada entre animales jóvenes (14.0 U/L) y adultos $(10.8 \mathrm{U} / \mathrm{L})$ puede considerarse normal.

La AST, una enzima de mayor versatilidad en la práctica médica equina, registró un valor que se halla dentro de rango referenciales (138-409 U/L) para la especie (Carlson, 2002), aunque ligeramente más altos que los reportados por Kaneko et al. (1997) para el equino (296 $\pm 70 \mathrm{U} / \mathrm{L})$. Es importante indicar que el número de animales jóvenes en la muestra puede alterar los promedios totales pues estos tienen a tener valores mayores de ALT por el mayor vigor y actividad muscular (Santamarina et al., 1994). Esta razón explicaría la diferencia significativa hallada entre animales jóvenes (355.6 U/L) y adultos (289.8 U/L).

El valor medio obtenido de ALP (60.0 $\mathrm{U} / \mathrm{L}$ ) fue considerablemente inferior a otros reportes ( $244 \pm 101$ U/L, Kaneko et al., 1997; 86-285 U/L, Carlson, 2002). Tal condición resulta difícil de explicar y podría atribuirse a un posible efecto artefacto de la hemólisis en la determinación de la enzima (Meyer y Harvey, 1998) o a la eficiencia del kit comercial empleado (Cardoso, 2008).

Los valores séricos de GGT (8.04 \3.24 $\mathrm{U} / \mathrm{L})$ fueron bastante similares a los citados para la especie equina $(7.6 \pm 1.5 \mathrm{U} / \mathrm{L})$ por Kaneko et al. (1997), aunque Carlson (2002) reporta un rango de valores superior (8-22 U/L). No hubo influencia del sexo y la edad en los valores de esta enzima, aunque hay estudios que señalan valores mayores en caballos jóvenes Cuarto de Milla debido a una masa hepática relativamente mayor como porcentaje del peso corporal (Gosset y French, 1984). Además, la raza es una causal de diferencia significativa y una razón válida para implementar un manejo clínico diferenciado (Lacerda et al., 2006). 
Los valores séricos de CK (437.54 \pm 129.3 U/L) resultaron ser considerablemente mayores a los citados por Kaneko et al. (1997), cuyo promedio es de $12.9 \pm 5.2 \mathrm{U} / \mathrm{L}$. Sin embargo, se encuentran más cercanos al rango de referencia referido por Carlson (2002), quien señala valores de 119-287 U/L. La razón más importante que podría explicar tal condición en este estudio es la actividad física a la que estuvieron sujetos algunos individuos como parte de su manejo diario, ya que el aumento de la actividad enzimática comprendida entre 400 y 500 U/L está relacionada al inicio del entrenamiento o en respuesta a un ejercicio moderado (Valberg and Hodgson, 2002). Este argumento se ve apoyado por los niveles relativamente altos de AST encontrados.

Los valores medios que describen la bilirrubinemia total, directa e indirecta se hallaron dentro de los rangos de referencia para la especie equina señalados por Kaneko et al. (1997) y Carlson (2002). Sin embargo, Kraft (1998) advierte que los valores medios normales de bilirrubina total son propios de cada raza; así, se reportan valores crecientes en el Poni Shetland ( $0.6 \mathrm{mg} / \mathrm{dL})$, razas de sangre fría $(1.2 \mathrm{mg} / \mathrm{dL})$ y razas de sangre caliente $(2.27 \mathrm{mg} / \mathrm{dl})$ (Ochoa y Vallenas, 1960; Blackmore y Brobst, 1981). Asimismo, las diferencias encontradas entre jóvenes y adultos podrían estar relacionadas al mayor número de eritrocitos en los jóvenes (Inope, 1960; Ochoa y Vallenas, 1960).

A pesar de que el análisis procura la revisión de los resultados sostenidos por diferentes autores, se debe considerar la disparidad de métodos y ausencia de estandarización de los métodos de laboratorio utilizados, la desigualdad en los criterios de selección de la muestra, así como el manejo y alimentación de los animales, de modo que no es factible observar con total objetividad las diferencias interraciales.

\section{Conclusiones}

? Los valores medios de los parámetros hemato-bioquímicos en ejemplares de la raza Caballo Peruano de Paso en aparente buen estado de salud se encontraron dentro del rango de referencia establecido para la especie, a excepción de los valores para las enzimas ALP y CK.

? El factor sexo no fue significativo, con excepción del recuento de monocitos y de la actividad sérica de la enzima CK $(\mathrm{p}<0.05)$.

? $\quad$ El factor edad fue significativo para 6 de las 13 variables hematológicas estudiadas (concentración de hemoglobina, los índices eritrocíticos VCM y CHCM, el recuento de eosinófilos y linfocitos, y la relación segmentados/linfocitos) y para 4 de las 8 variables bioquímicas (actividad sérica de las enzimas ALT y AST, y concentración de bilirrubina total y bilirrubina indirecta).

\section{Literatura Citada}

1. Andrews F, Geiserr D, White $S$, Williamson L, Maykuth P, Green E. 1995. Haematological and biochemical changes in horses competing in a 3 Star horse trial and 3-day-event. Equine Vet J Suppl 20: 57-63.

2. Bayly W. 1987. The interpretation of clinical pathologic data from the equine athlete. Vet Clin N Am Equine 3: 631647.

3. Benjamín M. 1991. Manual de patología clínica en veterinaria. México: Limusa. 421 p.

4. Blackmore D, Brobst D. 1981. Biochemical values in equine medicine. United Kingdom: Animal Health Trust. $108 \mathrm{p}$. 
5. Cardoso C. 2008. Comparação de kits comerciais na dosagem de constituintes bioquímicos do sangue em eqüinos hígidos. Tesis de Magister Scientiae. Minas Gerais, Brasil: Programa de PósGraduação em Medicina Veterinária, Univ. Federal de Vicoça. 123 p.

6. Carlson G. 2002. Clinical chemistry tests. In: Bradford P (ed). Large animal internal medicine. $3^{\text {rd }}$ ed. Chap 22. USA: Ed. Mosby. p 389-412.

7. Cebulj-Kadunc N, Božic M Kosec M, Cestnik V. 2002. The influence of age and gender on hematological parameters in Lipizzan horses. J Vet Med A 49: 217-221.

8. Coppo J, Mussart N. 2000. Apoyatura bioquímica al diagnóstico veterinario. Casuística registrada tras 25 años de funcionamiento de un servicio de análisis clínicos. Rev Vet 10: 34-39.

9. Craig L, Hintz H, Soderholm L, Shaw K, Schryver H. 1985. Changes in blood constituents accompanying exercise in polo horses. Cornell Vet 75: 297-302.

10. Deem D. 2002. Alterations in the erythron. In: Bradford P (ed). Large animal internal medicine. $3^{\text {rd }}$ ed. Chap 24. USA: Ed. Mosby. p 415 - 419.

11. García E. 2006. Caracterización morfológica, hematológica y bioquímica clínica en cinco razas asnales españolas para programas de conservación. Tesis doctoral. Barcelona: Facultad de Medicina Veterinaria, Univ Autónoma de Barcelona. $251 \mathrm{p}$.

12 Gómez C, Petrón P, Andaur M, Pérez $\boldsymbol{R}$, Matamoros $R$. 2004. Medición postejercicio de variables fisiológicas, hematológicas y bioquímicas en equinos de salto Holsteiner. Rev Cient 14: 244-253.

13. Gosset K, French D. 1984. Effect of age on liver enzyme activities in serum of healthy Quarter Horses. Am J Vet Res 45: 354-356.

14. Harvey R, Hambright M. 1985. Normal serum chemistry and hematology values of the American miniature horse. Equine Pract 7: 6-8.
15. Inope L. 1960. Algunos valores hematológicos en caballos pura sangre de carrera. Tesis de Médico Veterinario. Lima: Facultad de Medicina Veterinaria, Univ Nacional Mayor de San Marcos. 41 p.

16. Jain N. 1993. Essential of veterinary hematology. USA: Lea \& Febiger. 417 p.

17. Kaneko J, Harvey J, Bruss M. 1997. Clinical biochemistry of domestic animals. $5^{\text {th }}$ ed. USA: Academic Press. $932 \mathrm{p}$.

18. Kedzierski W, Bergero D. 2006. Comparison of plasma biochemical parameters in Thoroughbred and Purebred Arabian horses during the same-intensity exercise. Pol J Vet Sci 9: 233-238.

19. Kraft H. 1998. Métodos de laboratorio clínico en Medicina Veterinaria de mamíferos domésticos. $3^{\mathrm{a}}$ Ed. España: Acribia. $288 \mathrm{p}$.

20. Lacerda L, Campos $R$, Sperb $M$, Soares E, Barbosa P, Godinho E, Ferreira $R$, Santos V, González $F$. 2006. Hematologic and biochemical parameters in three high performance horse breeds from southern Brazil. Arch Vet Sci 11: 40-44.

21. Lording P. 2008. Erythrocytes. Vet Clin Equine 24: 225-237.

22. McFarlane D, Sellon D, Gibbs S. 2001. Age-relates quantitative alterations in lymphocyte subsets and immunoglobulin isotypes in healty horses. Am J Vet Res 62: 1413-1417.

23. Medeiros A, Dos Anjos $S$, Franciscato C, Segala L, Merina L. 2006. Valores hematológicos, proteínas plasmáticas totais e fibrinogênio do cavalo crioulo suas variações em relação ao sexo, idade e manejo. Acta Sci Vet 34: 275-279.

24. Meyer D, Harvey J. 1998. Laboratory medicine testing: specimen interferences and clinical enzymology. In: Meyer D, Harvey J (eds). Veterinary laboratory medicine. $2^{\text {nd }}$ ed. USA:. WB Saunders. p 3-22. 
25. Muñoz A, Lucas R, Benito M, Palacio J, López M, Satué K, Castejón F. 2001. Evaluación del entrenamiento mediante el análisis hematológico y bioquímico plasmático en caballos angloárabes de carreras. Med Vet 18: 491-499.

26. Ochoa J, Vallenas A. 1960. La bilirrubinemia en el suero sanguíneo de caballos pura sangre de carrera de nuestro medio. Rev Fac Med Vet 15: 145156.

27. Pérez R, García M, Cabezas I, Guzmán R, Merino V, Valenzuela S, González C. 1997. Actividad física y cambios cardiovasculares y bioquímicos del caballo chileno a la competencia de rodeo. Arch Med Vet 29(2): 221-234.

28. Riber C, Rubio M, Marquez F, Pinedo M, Munoz A, Castejon F. 1995. Haematological changes observed in Andalusian horses with laminitis. J Vet Med Sci 57: 981-984.

29. Risso J. 1994. Prólogo. En: Embajador silencioso - Orgullo del Perú. Tomo I. Lima: Ed. Gráfica Santa Fé. p i-iii.

30. Rodríguez-Gallardo P, Aguilar P, Vega J, Cara A. 1992. Frecuencias génicas de grupos sanguíneos y polimorfismos proteicos en el caballo de raza andaluza. Comparación con cuatro razas de caballos americanas. Arch Zootec 41: 433442.

31. Rossdale P, Ricketts S. 1980. Equine stud farm medicine. $2^{\text {d }}$ ed. London: Baillièrs Tindall. $564 \mathrm{p}$.

32. Santamarina G, Prieto F, Benedito J. 1994. El poni gallego: Hematología y bioquímica. España: Servicios de Publicaciones Diputación Provincial de Lugo, España. 311 p.

33. Schalm O, Jain N, Carroll E. 1975. Veterinary hematology. $3^{\text {rd }}$ ed. USA: Lea \& Febiger. 807 p.

34. Schalm O, Carlson G. 1982. The blood and blood-forming organs. In: Mansmann R, McAllister E, Pratt P (eds). Equine medicine $\&$ surgery. $3^{\text {rd }}$ ed. Vol I, Chap 11. USA: American Veterinary Publication. p 377-414.

35. Selvaraj P, Nambi A, Bhuvnakumar C, Dhanapalan P. 2008. Hepatic enzyme profile in Indian thoroughbred equines. Tamilnadu J Vet Anim Sci 4: 38-40.

36. Valberg S, Hodgson D. 2002. Diseases of muscle. In: Bradford P (ed). Large animal internal medicine. $3^{\text {d }}$ ed. Chap 40. USA: Mosby. p 1266-1291. 